ReVISTA de BIOLOGía TROPICAL

\title{
La pesca ilegal en el Parque Nacional Isla del Coco, Costa Rica
}

\author{
Andrés López-Garro ${ }^{1}$, Ilena Zanella ${ }^{1}$, Frank Martínez ${ }^{1}$, Geiner Golfín-Duarte ${ }^{2} \&$ \\ Maikel Pérez-Montero ${ }^{2}$ \\ 1. Asociación Conservacionista Misión Tiburón, Playas del Coco, Guanacaste, Costa Rica; alopez@misiontiburon.org, \\ izanella@misiontiburon.org, fmartinez1123@hotmail.com \\ 2. Parque Nacional del Coco, Área de Conservación Marina Isla del Coco, Sistema Nacional de Áreas de Conservación, \\ Costa Rica, geiner.golfin@sinac.go.cr, elcocolaisla@hotmail.com
}

Recibido 14-IV-2015. Corregido 21-VII-2015. Aceptado 31-VIII-2015.

\begin{abstract}
Illegal fishing at Isla del Coco National Park, Costa Rica. Isla del Coco National Park (ICNP), Costa Rica, is renowned for its large aggregations of pelagic species, especially sharks (Carcharhinidae and Sphyrnidae), billfish (Istiophoridae) and tuna (Scombridae). The high abundance of these sought after species, attracts commercial fishing vessels that enter the protected waters of the Park. Illegal fisheries thus represent one of the biggest threats to the ecological integrity of this World Heritage Site. The ICNP dedicates important resources to protection and control via regular surveillance patrols, along the 12 nautical miles that have Marine Protected Area (MPA) status. During 2012, 2013 and between January and August 2014, 405 surveillance patrols within the MPA found 108 fishing lines, more than 13000 hooks, seized more than $500 \mathrm{~km}$ of fishing line and 257 pelagic individuals. The yellowfin tuna (Thunnus albacares) was the most abundant species, representing more than $72.3 \%$ of the total catch; seized individuals had a mean total length of $153.35 \pm 23.19 \mathrm{~cm}$ (Min=90 cm; Max=190 cm); which indicates that most individuals were adults. Analysis of monthly CPUE showed that the highest relative abundance of illegally caught T. albacores was between May and August of each year, with a mean relative abundance of $26.13 \pm 54.71,11.63 \pm 23.79,13.71 \pm 24.52$ tunas per 1000 hooks in 2012, 2013, 2014, respectively. This species had a high survival rate (nearly $80 \%$ of the individuals found on the lines were still alive), probably due to the short fishing lines used by illegal fishermen, combined with the swift intervention of rangers. The CPUE of T. albacares found in this study is greater than records in Costa Rica's Exclusive Economic Zone ( 0.74 and 0.44 per 1000 hooks respectively). These results highlight the importance of ICNP as an aggregation site for T. albacares and the need for its effective protection. It is important that ranger intervention continue to be as rapid and effective as possible, in order to decrease the impact of illegal fishing on the marine biodiversity of the ICNP. Rev. Biol. Trop. 64 (Suppl. 1): S249-S261. Epub 2016 February 01.
\end{abstract}

Key words: Isla del Coco, Marine Protected Area, illegal fishery, Thunnus albacares, yellowfin tuna, Cocos Island.

La protección absoluta de un Área Marina Protegida (AMP) generalmente resulta en un rápido incremento en la biomasa, abundancia y talla promedio de las poblaciones explotadas (Roberts, Hawkins, \& Gell, 2005); además, produce un aumento significativo en la diversidad de especies (Halpern, 2003). Por esta razón, la creación de áreas de protección absoluta fomenta la recuperación de la biodiversidad marina y, a su vez, de los recursos marinos (Pauly et al., 2002). Sin embargo, a pesar de su reafirmada importancia, en el mundo las AMP abarcan menos del $1 \%$ de la superficie del océano y según Kelleher, Bleakley \& Wells (1995), son pocas las que brindan una protección efectiva de los objetos de conservación por lo que fueron creadas.

Al igual que la mayoría de las AMP en el mundo, las ubicadas en las islas oceánicas del Corredor Marino del Pacífico Tropical Oriental (Parque Nacional Isla del Coco (PNIC), Costa Rica; Parque Nacional Reserva Marina 
de Galápagos, Ecuador; Santuario de Flora y Fauna de Malpelo, Colombia) están siendo constantemente amenazadas por las pesquerías ilegales. Sobresalen embarcaciones dedicadas a la captura de atún aleta amarilla (Scombridae; Thunnus albacares) y tiburones (Carcharhinidae, Alopiidae y Sphyrnidae) con líneas de flote o palangre y redes de cerco (Bessudo et al., 2011).

El PNIC es un sitio con alta diversidad marina (Cortés, 2012) que posee una AMP con protección absoluta. El PNIC fue Declarado Sitio Patrimonio Natural de la Humanidad en 1997 por la Organización de Naciones Unidas para la Educación, la Ciencia y la Cultura (UNESCO, por sus siglas en inglés), por albergar hábitats esenciales para especies pelágicas migratorias, como tiburones (Carcharhinidae, Sphyrnidae), picudos (Istiophoridae) y atunes (Scombridae) (ACMIC, 2006). La presencia y abundancia de estas especies pelágicas en las aguas del PNIC y sus alrededores son la razón por la cual la incursión de embarcaciones pesqueras dentro del AMP es considerada una de las principales amenazas para la integridad de este Sitio Patrimonio Natural de la Humanidad (ACMIC, 2006). Para enfrentar esta problemática, el PNIC invierte diariamente recursos humanos y económicos en la protección de su AMP realizando patrullajes de control y vigilancia (ACMIC, 2006). Durante estos patrullajes es común los funcionarios decomisen o hallen equipos de pesca ilegal, los cuales además pueden tener organismos marinos (vivos o muertos) enganchados en sus anzuelos.

El número de embarcaciones que realiza faenas ilegales en el PNIC es variable a lo largo del año, sin embargo entre junio y diciembre se reporta la mayor cantidad de hallazgos y decomisos (Castro, Gonzáles, \& Estrada, 2008). Solo en el 2007, durante los patrullajes de vigilancia efectuados por la Comisión Tripartita (conformada por funcionarios de Servicio Nacional de Guardacostas, el Ministerio del Ambiente y Energía, y la Fundación MarViva), se observaron en total 1516 embarcaciones en las aguas del PNIC. Durante este año se lograron decomisar cerca de $600 \mathrm{~km}$ de líneas de pesca ilegales; en dichas líneas se encontraron 6674 anzuelos y un total de 193 especímenes marinos que correspondían a tiburones, atunes, picudos y tortugas marinas (Castro et al., 2008). Según Arias et al. (2014), entre el 2005 y el 2010 en el PNIC fueron halladas 300 líneas de pesca con aproximadamente 34500 anzuelos y 2000 organismos pelágicos enganchados, de los cuales el $66 \%$ eran atunes y $25 \%$ tiburones.

Hasta el 2010, durante los patrullajes de vigilancia, los funcionarios del PNIC solían registrar algunos datos básicos relacionados sobre todo a las maniobras operativas. Por lo anterior, la organización Misión Tiburón inició un proyecto para asesorar a los funcionarios del PNIC en la recolección de información técnica y científica biológica-pesquera. Como parte de esta iniciativa se desarrolló la presente investigación, cuyo fin es describir los artes de pesca hallados y las especies pelágicas capturadas por las embarcaciones que incursionaron ilegalmente en el PNIC durante el 2012, 2013 y entre enero y agosto del 2014.

\section{MATERIALES Y METODOS}

Área de Estudio: El PNIC se localiza en el Océano Pacífico $\left(5^{\circ} 30^{\prime}-5^{\circ} 34^{\prime} \mathrm{N}-87^{\circ} 01^{\prime}\right.$ $\left.87^{\circ} 06^{\prime} \mathrm{W}\right), 496 \mathrm{~km}$ al suroeste de Cabo Blanco, Costa Rica. El PNIC posee una extensión de $24 \mathrm{~km}^{2}$ en la parte terrestre y un área marina protegida con protección absoluta que se extiende 12 millas náuticas $(22.22 \mathrm{~km})$ alrededor de la Isla (Cortés, 2008) (Fig. 1). El PNIC pertenece al Área de Conservación Marina Isla del Coco (ACMIC), la cual además posee una zona contigua de alrededor de 1000000 ha denominada Área Marina de Manejo Montes Submarinos (AMM-MS). Los datos analizados sobre las pesquerías ilegales y especies capturadas provienen de líneas de pesca halladas dentro del PNIC durante el periodo 2012-2013 y entre enero y agosto del 2014.

Patrullajes de control y protección en el AMP del PNIC: Las condiciones oceanográficas, la disponibilidad de embarcaciones, personal, entre otras cosas hacen que el número de 


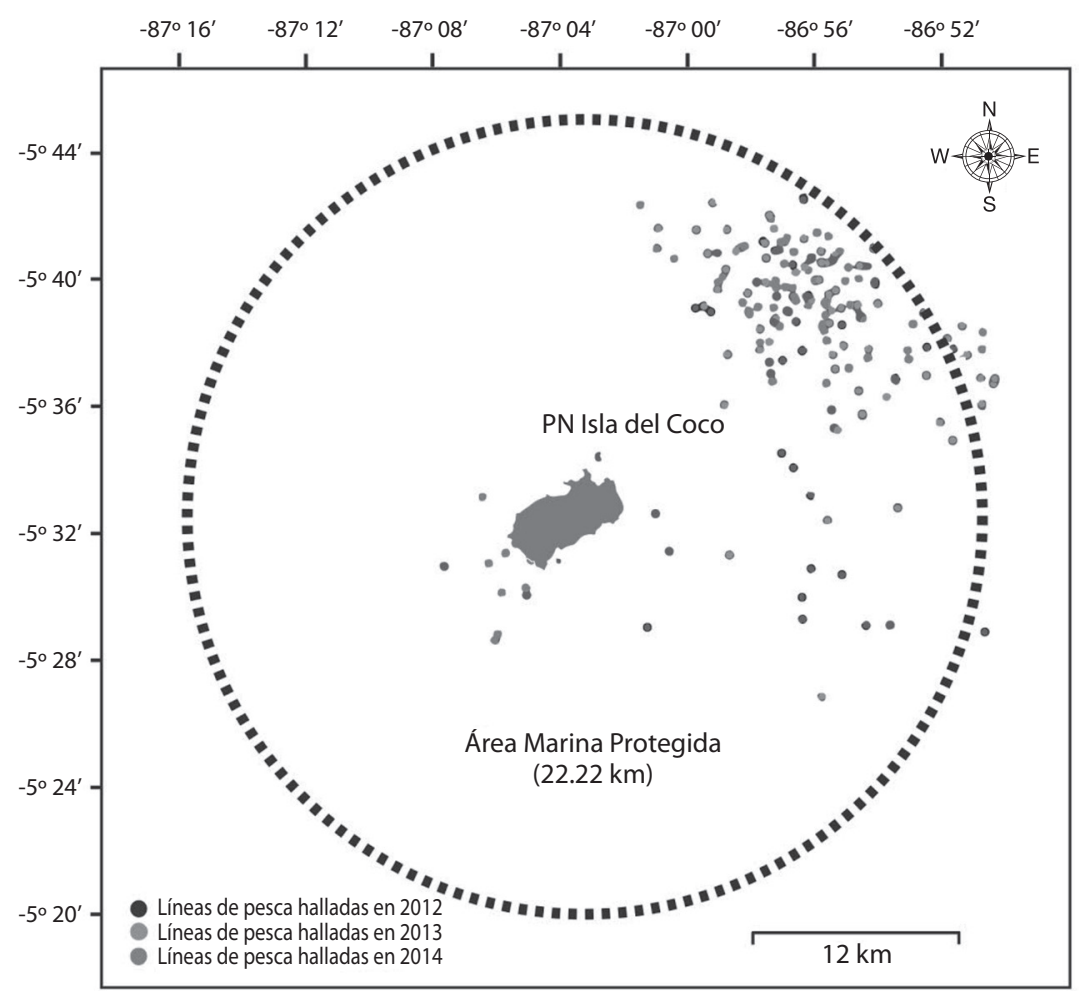

Fig. 1. Ubicación de las líneas de pesa ilegal encontradas de enero 2012 a agosto 2014, en el Área Marina Protegida del Parque Nacional Isla del Coco, Costa Rica.

Fig. 1. Geographic location of the illegal fishing operations found in the Marine Protected Area at Isla del Coco National Park, from January 2012 to August 2014).

patrullajes en el PNIC cambie mensualmente. De esta manera hay semanas donde se realizan patrullajes diarios, mientras hay otras donde no se patrulla.

Descripción de las pesquerías ilegales en el PNIC: Embarcaciones costarricenses nacionales y de otros países faenan de manera ilegal a las aguas protegidas del PNIC principalmente en busca de atunes ( $T$. albacares) y tiburones (Carcharhinidae y Alopiidae). Sobresalen embarcaciones de pesca comercial de mediana y avanzada escala costarricenses que utilizan la línea de flote o palangre como arte de pesca (Cajiao, 2005). Sin embargo, es común observar barcos semi-industriales de otros países con redes de cerco, que buscan capturar atunes y otros pelágicos comerciales en los alrededores del PNIC (Serna, 2006).
Los equipos de pesca que son hallados en el PNIC son conocidos como palangre o "long-line" y constan de una línea madre de monofilamento de varios kilómetros de largo, de la que cuelgan líneas secundarios llamada "bajantes" con anzuelos tipo circulares $\left(\mathrm{N}^{\mathrm{o}} 14\right.$, 15 y 16) y "J" taiwanés ( ${ }^{\circ} 3$ y 4 ) aproximadamente cada $25-40 \mathrm{~m}$. Por lo general, las líneas son lanzadas en horas de la noche y madrugada. La carnada que utilizan los pescadores normalmente consiste de calamares (Loliginidae), tiburones (sobre todo tiburones zorros (Alopias pelagicus), y atunes negros (Scombridae). Además algunos equipos de pesca son hallados con carnada viva, sobre todo utilizan peces chanchos pelágicos de la familia Balistidae.

Información pesquera y biométrica: La información pesquera referente a los artes de 
pesca y especies pelágicas encontrados en el AMP del PNIC y alrededores fue recopilada entre enero 2012 y agosto 2014. Para cada una de las líneas de pesca se tomaron las coordenadas geográficas del inicio y final donde esta fue hallada y recolectada. Además se tomó información sobre el número de anzuelos, la carnada, el largo de la línea utilizada, el tipo de anzuelo, y la especie objetivo. Con respecto a las especies halladas en las líneas de pesca se efectuó un registro de la captura por especie y su condición (vivo o muerto) al recoger la línea de pesca. Con las posiciones geográficas de las líneas halladas se realizó un mapa; además se calculó la longitud de las líneas, y el promedio, mínimo y máximo de anzuelos en las líneas de pesca halladas. Además, se graficó mensualmente el número de líneas halladas.

Todos los individuos hallados fueron identificados hasta el nivel especie y agrupados en cinco categorías: peces pelágicos (incluye peces óseos de la familia Coryphaenidae, Istiophoridae y Scombridae), tiburones (incluye tiburones de la familias: Alopiidae, Sphyrnidae y Carcharhinidae), tortugas marinas, manta rayas y delfines. Además, se calcularon los porcentajes aportados por cada categoría y se liberaron aquellos individuos encontrados con vida. Para los especímenes hallados muertos, cuando las condiciones lo permitieron, se recolectaron datos biométricos como la Longitud Total (LT: medida desde la punta del hocico, hasta el extremo final de la aleta caudal, $\pm 0.1 \mathrm{~cm})$, y en la especie más abundante $T$. albacares se calculó la proporción sexual, la longitud total media, así como la desviación estándar (DE), mínimos y máximos.

Para los individuos de T. albacares además se realizó una evaluación interna de las gónadas a fin de evaluar el sexo y estadío reproductivo. Se utilizó la prueba Chi-cuadrado para evaluar diferencias en las proporciones de machos y hembras de atún aleta amarilla. Para la evaluación del estadío reproductivo de los T. albacares, se utilizó una readecuación de la clasificación de madurez macroscópica utilizada para el atún aleta azul (Thunnus thynnus) en el Atlántico Oeste (Knapp, Heinisch1, Rosenfeld, \& Lutcavage, 2013).

\section{MACHOS}

Estadío I: Inmaduro. Testes flácidos y delgados de color amarillento o transparente.

Estadío II: Madurando. Testes engrosando, coloración blancuzca o amarillenta.

Estadío III: Maduro. Testes muy agrandados y blancuzcos, con presencia de esperma.

\section{HEMBRAS}

Estadío I: Inmaduro. Gónadas flácidas y delgadas de color amarillento o anaranjado.

Estadío II: Madurando. Gónadas engrosando, oocitos visibles amarillentos o ligeramente anaranjados.

Estadío III: Maduro. Gónadas muy agrandadas con oocitos maduros de coloración rojizo-amarilla. Presencia de mucha irrigación (vascularización) en la gónada.

Abundancia Relativa: La abundancia relativa para la especie más capturada, $T$. albacares, fue estimada mediante el cálculo de la Captura por Unidad de Esfuerzo (CPUE), índice de abundancia relativa que permite comparaciones espacio-temporales. La CPUE se estimó a partir del número de especímenes capturados por cada 1000 anzuelos hallados durante el 2012. El bajo número de líneas halladas en algunos meses del 2013 y 2014 no permitió estimar la CPUE en estos años.

\section{Patrullajes realizados y líneas de pesca} halladas: Se estimó el promedio de patrullajes mensuales, así como la desviación estándar (DE), mínimos y máximo. Por medio de regresiones simples, se relacionó la abundancia relativa (CPUE) mensual (variable dependiente) y el número de patrullajes realizados (variable independiente), así como la abundancia relativa (CPUE) (variable dependiente) y las líneas de pesca halladas (variable independiente), utilizando el programa InfoStat (Di Rienzo et al., 2008). 


\section{RESULTADOS}

En total se realizaron 405 patrullajes en el AMP y en aguas del AMM-MS entre el 20122014 para un promedio de $12.2 \pm 3.9$ patrullajes por $\operatorname{mes}(\min =4$ y $\max =19)$. De estos los meses con mayor cantidad de patrullajes son marzo y abril, donde se realizaron entre el 2012 y 2014 un total de $56(13.8 \%)$ y 48 (11.9\%) patrullajes respectivamente. A su vez, noviembre y diciembre fueron meses en donde los funcionarios realizaron $7(1.8 \%)$ y $15(3.8 \%)$ patrullajes respectivamente entre el 2012 y 2014.

Durante los patrullajes se encontraron 108 líneas de pesca ilegal a la deriva, representando $503.8 \mathrm{~km}$ de palangre ilegal y 12906 anzuelos (Cuadro 1). La mayoría de estas (cerca del 90 $\%$ ) fueron halladas al noreste de la Isla del Coco entre los 12 y $20 \mathrm{~km}$ dentro del AMP del PNIC. Menos del $10 \%$ de las líneas halladas fueron ubicadas al sur de la Isla del Coco (Fig. 1). En promedio, las 108 líneas de pesca halladas en el AMP presentaron un largo de $4.70 \pm 3.14 \mathrm{~km}$. El número de anzuelos por línea varió entre 6 y 349 , con un promedio de $123 \pm 16.5$ anzuelos.

Aunque hubo una presencia constante de embarcaciones pesqueras durante todo el periodo de estudio, fue en los meses lluviosos (abrilsetiembre) donde se halló el mayor número de líneas de pesca. Tal es el caso del 2012, año en que más del $70 \%$ de las líneas de pesca se halló entre julio y setiembre. A su vez, durante el 2013, más del $60 \%$ del total de líneas de pesca fueron halladas entre mayo y setiembre. De la misma forma en el 2014 los meses con mayores hallazgos fueron entre abril y agosto, meses lluviosos en los cuales se encontró más $90 \%$ de las líneas de pesca (Fig. 2).

Los peces pelágicos fueron el grupo de mayor captura, representado el $79.3 \%(n=212)$

\section{CUADRO 1}

Artes de pesca hallados en el Área Marina Protegida del Parque Nacional Isla del Coco, de enero 2012 a agosto 2014

TABLE 1

Fishing gear found in the Marine Protected Area at Isla del Coco National Park between January 2012 and August 2014

\begin{tabular}{lcccc}
\multicolumn{1}{c}{ Patrullajes } & 2012 & 2013 & Enero-Agosto 2014 & Totales \\
Total de patrullajes realizados & 160 & 135 & 110 & 405 \\
Total de líneas halladas & 47 & 38 & 23 & 108 \\
Total de línea halladas $(\mathrm{km})$ & 205.8 & 197.7 & 100 & 503.5 \\
Largo promedio líneas halladas (km) & $4.38 \pm 3.80$ & $5.32 \pm 3.12 \mathrm{DE}$ & $4.4 \pm 2.5 \mathrm{DE}$ & $4.7 \pm 3.14 \mathrm{DE}$ \\
Número total de anzuelos hallados & 4986 & 5261 & 2659 & 12906 \\
Número promedio de anzuelos x línea & $106.10 \pm 71.5$ & $138.8 \pm 74.8$ & $126.7 \pm 73.6$ & $123.9 \pm 16.5$ \\
Total de boyas (Galones y plásticas) & 1627 & 1538 & 782 & 3947 \\
Total de organismos pelágicos hallados & 130 & 76 & 51 & 257 \\
\hline
\end{tabular}

\section{CUADRO 2}

Porcentaje de individuos por categorías hallados en las líneas de pesca ilegal en el en el Área Marina Protegida del Parque Nacional Isla del Coco de enero 2012 a agosto 2014

TABLE 2

Percentage of individuals by species caught by illegal fishing lines found in the Marine Protected Area at Isla del Coco National Park between January 2012 and August 2014

\begin{tabular}{lcccc}
\multicolumn{1}{c}{ \% Categoría } & 2012 & 2013 & Enero-Agosto 2014 & Promedio \\
Peces pelágicos & 88.46 & 82.89 & 66.67 & 79.34 \\
Tiburones & 6.92 & 15.79 & 31.37 & 18.03 \\
Tortugas marinas & 3.85 & 1.32 & 0.00 & 1.72 \\
Mantas & 0.77 & 0.00 & 0.00 & 0.26 \\
Delfines & 0.00 & 0.00 & 1.96 & 0.65 \\
\hline
\end{tabular}




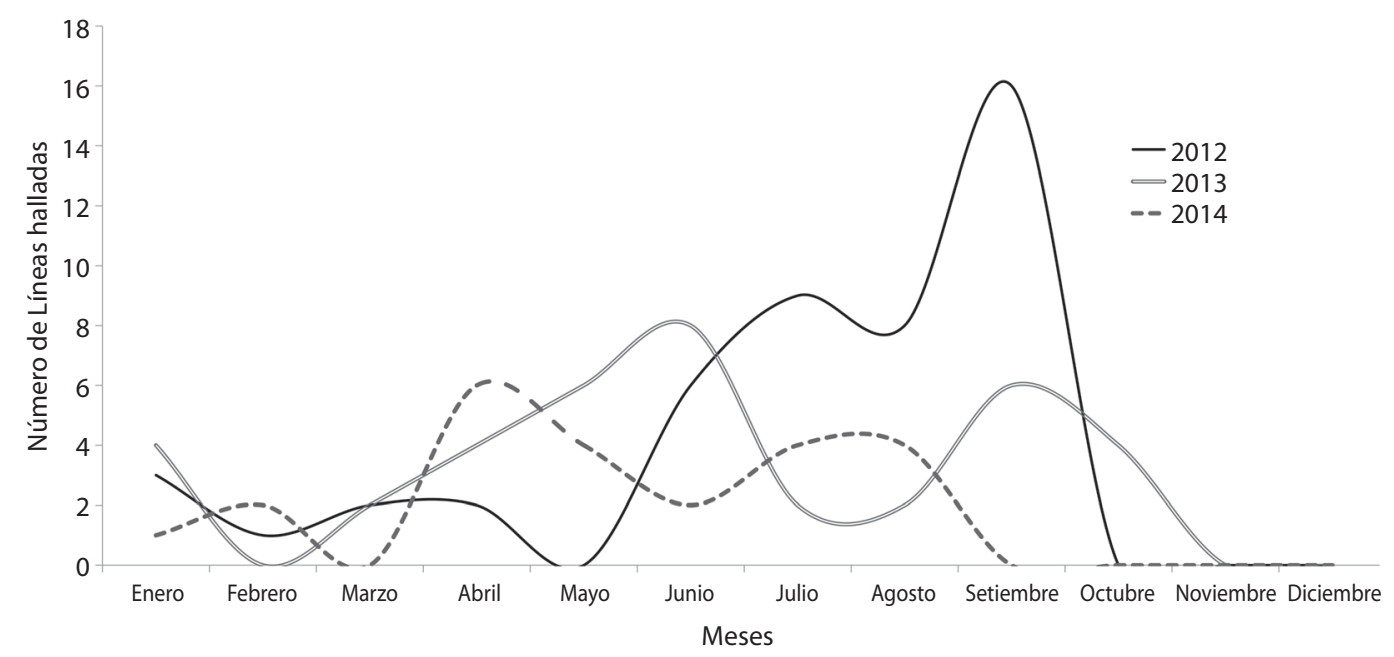

Fig. 2. Líneas de pesca ilegal halladas en el Área Marina Protegida del Parque Nacional Isla del Coco, de enero 2012 a agosto 2014 .

Fig. 2. Illegal fishing lines found per month in the Marine Protected Area at Isla del Coco National Park January 2012 to August 2014.

de todos los individuos analizados durante el periodo de estudio (2012-2014) (Cuadro 2). En esta categoría se incluye $T$. albacares $(\mathrm{n}=186)$, los atunes barriletes (Sarda Orientalis, $\mathrm{n}=4$, y Euthynnus lineatus, $\mathrm{n}=6$ ), el dorado (Coryphaena hippurus, $\mathrm{n}=4$ ), el pez vela (Istiophorus platypterus, $\mathrm{n}=3$ ), el atún negro (Katsuwonus pelamis, $\mathrm{n}=3$ ), el marlín negro (Makaira indica, $\mathrm{n}=2$ ), el pez luna (Mola mola, $\mathrm{n}=2$ ), la cabrilla (Epinephelus sp., $\mathrm{n}=1$ ) y el wahoo (Acanthocibium solandri, $\mathrm{n}=1$ ). El 18.0 $\%$ de los individuos capturados $(\mathrm{n}=37)$ en las líneas de pesca incluyeron varias especies de tiburones, de los cuales 21 individuos fueron tiburones sedosos (Carcharhinus falciformis), 7 tiburones martillo (Sphyrna lewini), 7 tiburones zorro (Alopias pelagicus) y 2 tiburones tigre (Galeocerdo cuvieri) (Cuadro 3).

Para T. albacares, el $31.7 \%$ de los individuos fueron hallados muertos. Las condiciones ambientales permitieron recolectar datos de talla y sexo de un total de 45 individuos de T. albacares. De estos el $54.5 \%$ fueron hembras. No hubo diferencias en la proporción de machos a hembras $\left(\chi^{2}=0.09 ; \mathrm{p}>0.05\right)$. Los análisis reproductivos efectuados en $45 \mathrm{~T}$. albacares hallados muertos en las líneas de pesca revelan que el $90 \%$ de estos experimentaba un avanzado estado de desarrollo gonadal (estadío reproductivo III), esto tanto en hembras como los machos.

El tamaño de los atunes $T$. albacares varió entre 90-190 cm LT, con una talla promedio de $153.35 \pm 23.19 \mathrm{~cm}$ LT. La Figura 3 muestra la distribución de frecuencia por longitud total de los atunes $T$. albacares analizados durante los hallazgos en el AMP. La mayoría de estos (>55\%), tenía un tamaño entre los 161 y $180 \mathrm{~cm}$ LT. A su vez, cerca del $25 \%$ de T. albacares analizados presentaron tallas entre los 131 y $150 \mathrm{~cm}$ LT, mientras que un $15 \%$ las tallas variaron entre entre los 90 y $120 \mathrm{~cm} \mathrm{LT} \mathrm{(Fig.} \mathrm{3).}$

Para T. albacares, las mayores capturas se presentaron entre mayo y agosto de cada año. Los análisis de Captura por Unidad de Esfuerzo (CPUEx1 000 anzuelos) revelan una abundancia relativa promedio anual para el 2012 de $26.13 \pm 54.71$ atunes (T. albacares) por cada 1000 anzuelos hallados. Mientras que en 2013 la CPUE fue de $11.63 \pm 23.79$ atunes cada 1000 anzuelos y en el 2014 de $13.71 \pm 24.52$ atunes cada 1000 anzuelos (Fig. 4).

Al graficar la CPUE mensual promedio de $T$. albacares con el número de líneas de 
CUADRO 3

Total de individuos por especie hallados en las líneas de pesca ilegal en el en el Área Marina Protegida del Parque Nacional Isla del Coco, de enero 2012 a agosto 2014

TABLE 3

Total of individuals by species caught by illegal fishing lines found in the Marine Protected Area at Isla del Coco National Park between January 2012 and August 2014

\begin{tabular}{lccccc} 
& 2012 & 2013 & Enero-Agosto 2014 & Totales 2012-2014| & \% del total \\
Atún aleta amarilla (Thunnus albacares) & 98 & 55 & 33 & 186 & 72.4 \\
Atún negro (Katsuwonus pelamis) & 2 & 0 & 1 & 3 & 1.2 \\
Barrilete (Sarda orientalis) & 4 & 0 & 0 & 4 & 1.6 \\
Barrilete negro (Euthynnus lineatus) & 6 & 0 & 0 & 6 & 2.3 \\
Cabrilla (Epinephelus sp.) & 1 & 0 & 0 & 1 & 0.4 \\
Delfin nariz de botella (Tursiops truncatus) & 0 & 0 & 1 & 1 & 0.4 \\
Dorado (Coryphaena hippurus) & 2 & 2 & 0 & 4 & 1.6 \\
Manta gigante (Manta birostris) & 1 & 0 & 0 & 1 & 0.4 \\
Marlin negro (Makaira indica) & 1 & 1 & 0 & 2 & 0.8 \\
Pez luna (Mola mola) & 0 & 2 & 0 & 3 & 0.8 \\
Pez vela (Istiophorus platypterus) & 0 & 3 & 0 & 7 & 1.2 \\
Tiburón martillo común (Sphyrna lewini) & 2 & 2 & 3 & 21 & 2.7 \\
Tiburón sedoso (Carcharhinus falciformis) & 0 & 9 & 12 & 2 & 8.2 \\
Tiburon tigre (Galeocerdo cuvieri) & 1 & 0 & 1 & 7 & 0.8 \\
Tiburón zorro Común (Alopias pelagicus) & 6 & 1 & 0 & 6 & 2.7 \\
Totuga verde o negra (Chelonia mydas) & 5 & 1 & 0 & 1 & 2.3 \\
Wahoo (Acanthocybium solandri) & 1 & 0 & 0 & $\mathbf{2 5 7}$ & 0.4 \\
Totales & $\mathbf{1 3 0}$ & $\mathbf{7 6}$ & $\mathbf{5 1}$ & $\mathbf{1 0 0}$ \\
\hline
\end{tabular}

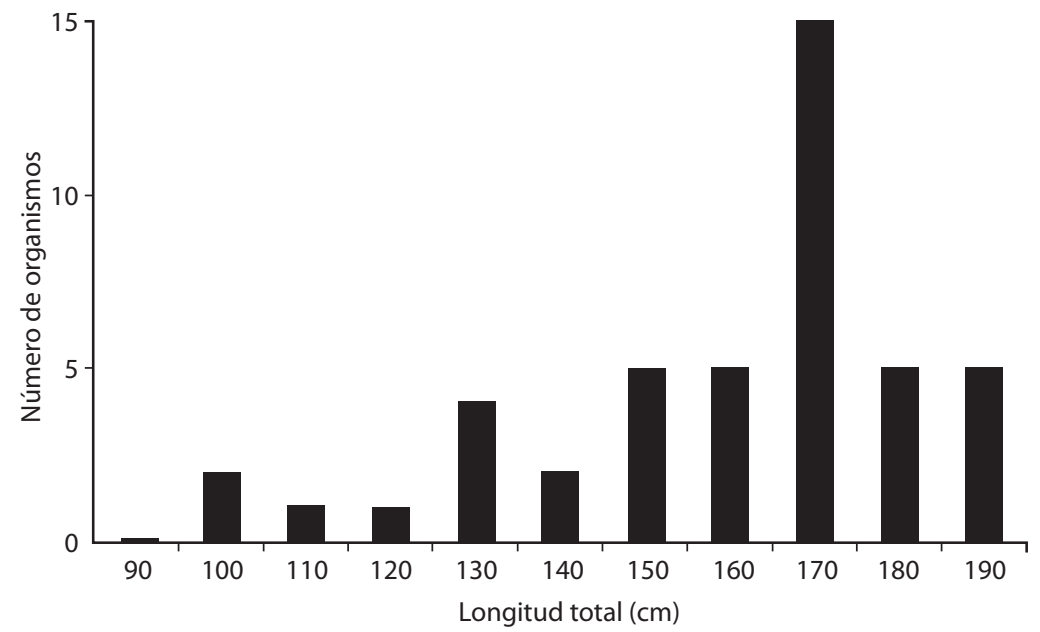

Fig. 3. Distribución de frecuencia por Longitud Total $(\mathrm{LT}=\mathrm{cm})$ de los atunes (T. albacares) analizados durante hallazgos de líneas de pesca ilegal en el en el Área Marina Protegida del Parque Nacional Isla del Coco, de enero 2012 a agosto 2014. Fig. 3. Frequency distribution for total length $(\mathrm{LT}=\mathrm{cm})$ of yellowfin tuna (T. albacares) analyzed during findings of illegal fishing's lines in the Marine Protected Area at Isla del Coco National Park from January 2012 to August 2014. 


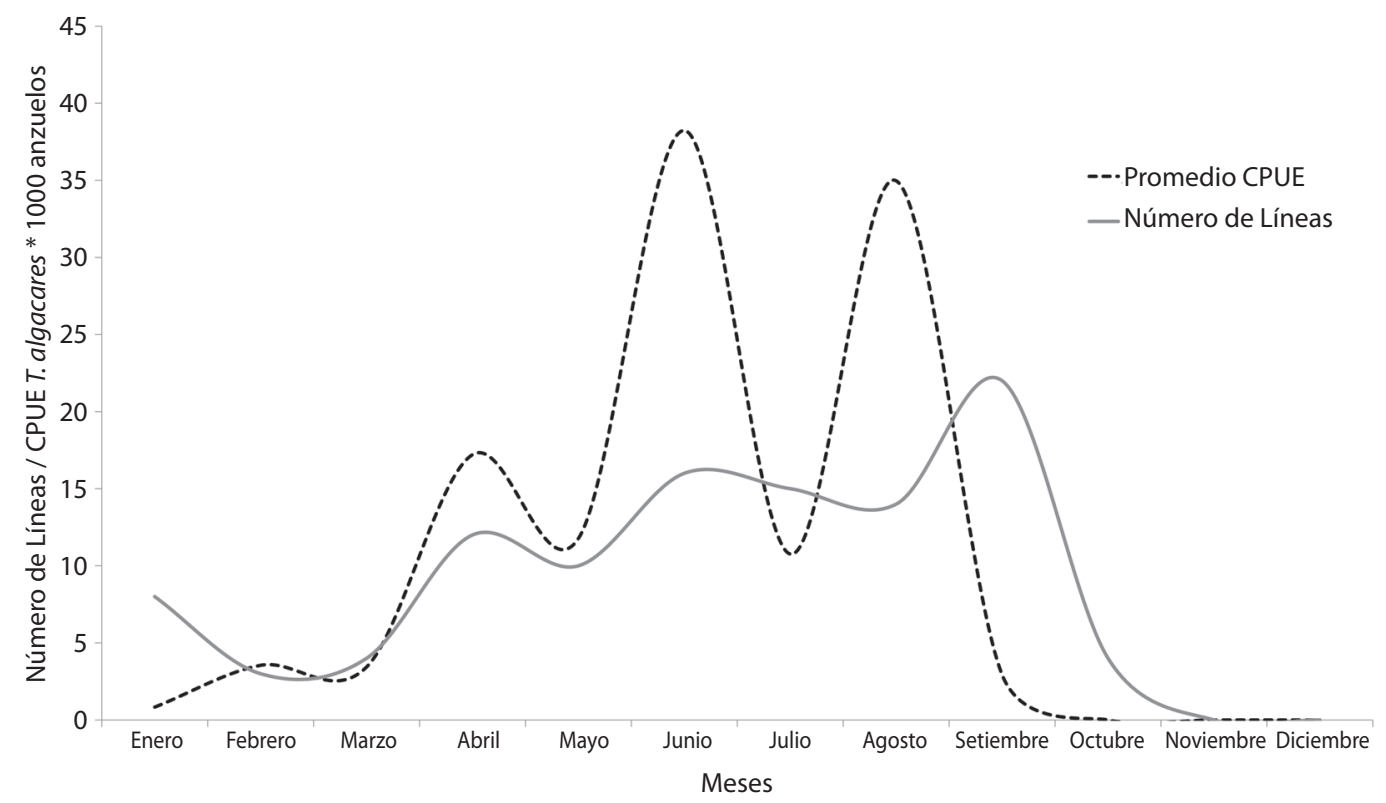

Fig. 4. Número de líneas halladas y captura por unidad de esfuerzo (CPUE) del atún aleta amarilla (Thunnus albacares) mensualmente en el Área Marina Protegida del Parque Nacional Isla del Coco, de enero 2012 a agosto 2014.

Fig. 4. Number of lines found and monthly catch per unit effort (CPUE) of yellow fin tuna (Thunnus albacares) in the Marine Protected Area at Isla del Coco National Park, between January 2012 and August 2014.

pesca halladas se observa que los meses donde se obtuvieron las mayores CPUE de T. albacares coinciden con los meses donde fueron halladas más líneas de pesca dentro del PNIC (Fig. 5). Sin embargo, se determinó que la CPUE mensual de T. albacares no depende del número de líneas de pesca halladas $\left(\mathrm{R}^{2}=0.30\right.$; $\mathrm{p}=0.06$ ), ni del número de patrullajes realizados $\left(\mathrm{R}^{2}=0.08 ; \mathrm{p}=0.39\right)$.

\section{DISCUSIÓN}

Su ubicación en aguas oceánicas convierte al PNIC en un lugar de una riqueza marina excepcional, lo anterior en gran parte influenciado por diferentes corrientes y la presencia de varios montes submarinos en sus alrededores (Cortés, 2012; Lizano, 2012). A su vez, esta alta biodiversidad y riqueza marina convierten al PNIC en un lugar muy atractivo para los pescadores. Esto lo confirman los hallazgos de líneas de pesca ilegal dentro del AMP entre 2012 y 2014, periodo durante el cual se encontraron más de $500 \mathrm{~km}$ de líneas de pesca y cerca de 13000 anzuelos.

Las líneas de pesca fueron halladas en diferentes zonas del AMP del PNIC; sin embargo, más del $80 \%$ de estas se encontraron al noreste de la Isla del Coco, a una distancia variable entre los 12 y $20 \mathrm{~km}$ de la costa (Fig. 1). Esto concuerda con lo reportado recientemente por Lizano (2012), quien describió un monte submarino ubicado a $14.8 \mathrm{~km}$ al noreste de la Isla del Coco. Los montes submarinos producen alteraciones en el flujo de las corrientes, creando surgencias ricas en nutrientes que favorecen el crecimiento del fitoplancton y de la biomasa de consumidores (desde zooplancton hasta depredadores tope) (Klimley, Jorgensen, Melo, \& Beavers, 2003); esto atrae a su vez a grandes pelágicos, como atunes y tiburones. De hecho, algunos montes submarinos son importantes sitios de agregación para especies pelágicas altamente migratorias (Morato, Hoyle, \& Nicol, 2010). 


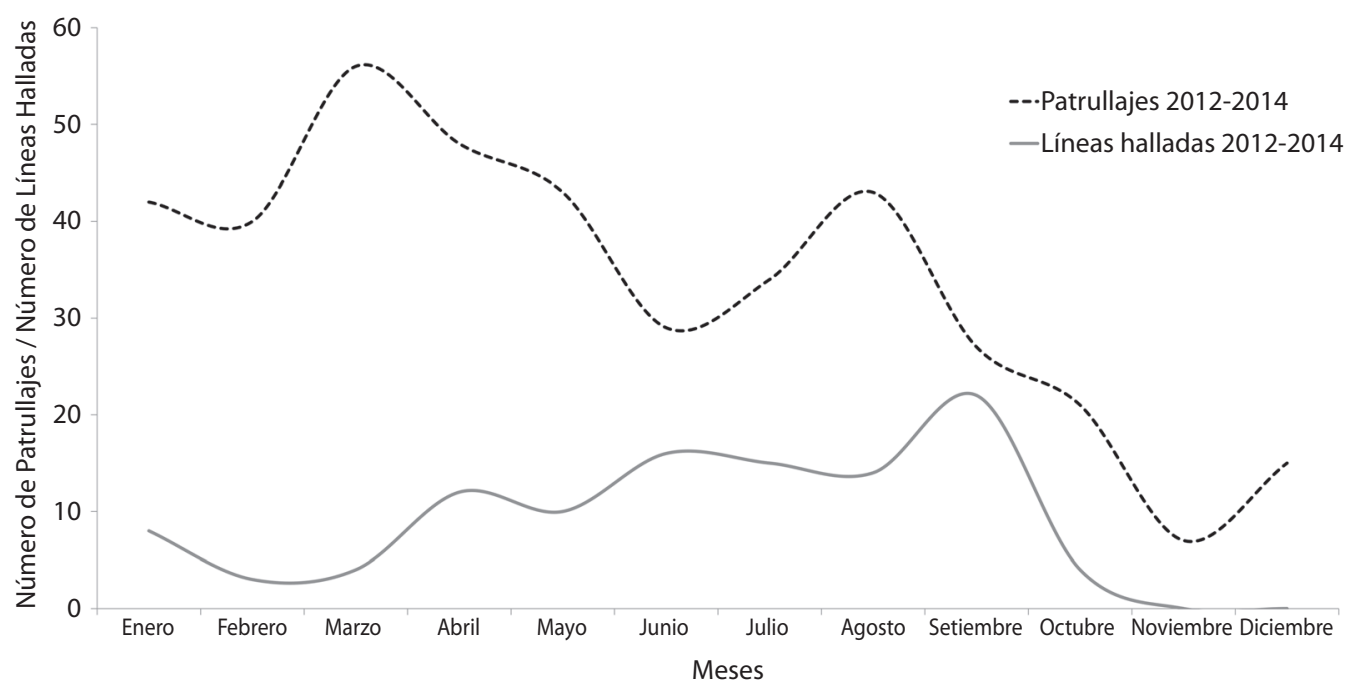

Fig. 5. Número de líneas halladas y de patrullajes realizados por mes en el Área Marina Protegida del Parque Nacional Isla del Coco, de enero 2012 a agosto 2014.

Fig. 5. Number of lines found and monthly catch per unit effort (CPUE) of yellow fin tuna (Thunnus albacares) in the Marine Protected Area at Isla del Coco National Park, between January 2012 and August 2014.

En el Pacífico Tropical Central y Occidental, Morato et al. (2010), utilizaron datos de observación a bordo de faenas de pesca con palangre para investigar el rol de los montes submarinos en la agregación de grandes pelágicos, concluyendo que los montes submarinos son "puntos caliente" de biodiversidad pelágica. Según Morato et al. (2010), existen una mayor riqueza de especies pelágicas asociadas a montes submarinos que en áreas costeras $u$ oceánicas. Según Klimley et al. (2003) dichas agregaciones se pueden producir por distintos motivos, como alimentación o reproducción. De esta manera, los montes submarinos generan condiciones que incrementan el flujo vertical de nutrientes, promoviendo la productividad en general y atrayendo especies pelágicas. Morato et al. (2010) identificaron diferentes especies cuyas capturas pesqueras aumentaron con la presencia de montes submarinos. Tal es el caso del tiburón mako (Isurus oxyrinchus) el tiburón sedoso (C. falciformis), los marlin (Makaira sp.) y los atunes aleta amarilla (T. albacares), entre otros.

Las líneas de pesca halladas durante los patrullajes realizados en el periodo de estudio, refleja que los pescadores que ingresan al AMP del PNIC utilizan principalmente líneas que abarcan cortas distancias $(2-4 \mathrm{~km})$ provistas de un bajo número de anzuelos (120-130 anzuelos). Esto las diferencia de las líneas de palangre utilizadas normalmente por la flota nacional de avanzada y mediana escala (Cajiao, 2005) que operan en aguas de la ZEE costarricense, estas embarcaciones utilizan líneas de pesca que abarcan entre 35-80 km de largo y constan de 804 \pm 94 anzuelos (Swimmer, Wang, Arauz, \& McCracken, 2010; Swimmer et al., 2011). Esta gran diferencia en el tamaño de las líneas y por ende del esfuerzo pesquero, se debe sobre todo al alto riesgo asociado a utilizar sus artes de pesca en un área protegida, ya que perder las líneas tiene un valor económico significativo y recoger líneas más largas conlleva mayor tiempo para evadir los controles.

Aunque hubo avistamientos de embarcaciones pesqueras y patrullajes durante todos los meses del año, los análisis muestran que la mayoría de líneas fueron halladas durante la época lluviosa (mayo-octubre), esto sin importar la cantidad de patrullajes realizados. En julio, agosto y setiembre se encontró más 
del $35 \%$ de las líneas de pesca analizadas, mientras el mayor número de patrullajes se realizó en marzo y abril con respectivamente $56(13.8 \%)$ y $48(11.9 \%)$.

Esto sugiere un aumento de la pesca ilegal durante los meses de época lluviosa, lo cual probablemente esté relacionado a una mayor concentración de nutrientes en las aguas del PNIC, que produce un aumento en la biomasa de consumidores, como atunes y tiburones (Serna, 2006). Además, a partir de mayo inicia a manifestarse la Contracorriente Ecuatorial Norte (CCEN), la cual llega a establecerse durante el tercer trimestre del año (julio-agosto-setiembre), corriente que atraviesa a la Isla del Coco en su paso hacia Suramérica (Lizano, 2008). La influencia de la CCEN en Isla del Coco puede ser la que explique el aumento de los hallazgos y de la abundancia relativa de especies pelágicas en el PNIC durante los meses más lluviosos del año.

A su vez, en noviembre y diciembre no se encontraron líneas de pesca dentro del AMP del PNIC. Lo anterior posiblemente se debe a que durante estos meses, los pescadores costarricenses de palangre dirigen sus esfuerzos a otras especies pelágicas estacionales y de importante valor comercial como lo es el dorado (C. hippurus). El dorado es una especie migratoria costera capturada en grandes cantidades a lo largo del Pacífico Oriental Tropical, con patrones de estacionalidad reportados por distintos autores. En Costa Rica, en los años noventas Campos, Segura, Lozano \& Madrigal (1993) reportaron un aumento de sus capturas entre setiembre y febrero. Mientras que en Panamá, las mayores capturas se reportaron durante los tres primeros meses del año (eneromarzo) (Lasso, \& Zapata, 1999). En el Pacífico de Ecuador, la temporada de pesca del dorado se registra entre noviembre y mayo, siendo los meses de mayor abundancia diciembre, enero y febrero (Patterson, \& Martínez, 1991).

Los análisis de las especies halladas en las líneas de pesca muestran que más del $70 \%$ de los organismos capturados fueron atunes aleta amarilla. De hecho T. albacares fue la especie más abundante en el 2012 (75,4 \%), 2013
$(72,4 \%)$ y de enero a agosto del 2014 (64,5 $\%)$. El alto valor comercial de esta especie en el mercado internacional, es sin duda la razón por la cual los pescadores ingresan permanente al AMP del PNIC. Es importante mencionar que durante los años 2013-2014 hubo un leve aumento en la captura de tiburones, sobre todo del tiburón sedoso (C. falciformis).

Con respecto a la condición de captura de los atunes T. albacares hallados en las líneas de pesca, la mayoría fueron encontrados vivos $(68,3 \%)$. Esto sugiere una rápida intervención del personal de control y protección del PNIC, ya que la mayoría de especies pelágicas requiere de altos niveles de oxígeno para sobrevivir, los cuales están ligados a una constante natación que se imposibilita al ser capturados en las líneas (Davis, 2002; Gale, Hinch, \& Donaldson, 2013). Sin embargo, las variables que determinan el tiempo que pueden sobrevivir un espécimen capturado por una línea de pesca son diversas como la temperatura del agua, profundidad, tiempo y enganche de captura, tamaño y físiología de las especies, entre otras. La interacción entre estos factores es poco entendida particularmente en escenarios de pesca incidental (Gallagher, Orbesenc, Hammerschlaga, \& Serafy, 2014). Información como la anterior no solo explica por qué algunas especies son halladas con vida, si no también pueden ser utilizadas como base para manejar futuras pesquerías en el AMM-MS y así minimizar la captura incidental de especies de tiburones en peligro de extinción como $S$. lewini (Gallagher et al., 2014).

Los atunes hallados muertos en las líneas permitieron recolectar valiosa información sobre la población de esta especie que habita en las aguas del PNIC. Con una población de proporciones sexuales similares (1:1.15), los atunes hallados presentaron una LT promedio mayor al $1.5 \mathrm{~m}$, lo cual nos indica que la mayoría de los individuos eran adultos. Lo anterior debido a que, para T. albacares, las tallas de primera madurez sexual reportadas oscilan entre los 90 y $120 \mathrm{~cm}$ (Albaret, 1977; Koido, \& Suzuky, 1989). Así mismo, los análisis reproductivos revelan que la mayoría de 
los organismos contenían gónadas en avanzado estado de madurez. Por lo anterior, es importante dar seguimiento al análisis reproductivo de los individuos capturados, esto con el fin de determinar si zonas específicas del PNIC son utilizadas como área de desove para T. albacares.

\section{SUGERENCIAS PARA LA CONSERVACIÓN Y LA INVESTIGACIÓN EN EL PNIC:}

1. Más del $80 \%$ de las líneas de pesca halladas durante los patrullajes se registró al noreste del PNIC, entre los 12 y $20 \mathrm{~km}$ de distancia de la Isla del Coco. La gran cantidad de líneas halladas en esta zona y su cercanía con los límites de la AMP, hace necesario recomendar a los administradores del Área de Conservación Marina Isla del Coco (ACMIC) considerar el diseño de una zona de amortiguamiento que se extienda al noreste del PNIC dentro del Área Marina de Manejo Montes Submarinos (AMM-MS). En dicha zona se recomienda prohibir o limitar la pesca con línea de flote o palangre, esto con el fin de prevenir la entrada ilegal de embarcaciones pesqueras al PNIC y conservar la integridad ecológica de esta área.

2. El atún aleta amarilla (T. albacares) resultó ser la especie más capturada por las líneas de pesca ilegal halladas en el PNIC, siendo probablemente la especie más buscada por los pescadores por su alto valor comercial. Por tanto, se recomienda realizar estudios a largo plazo con el fin de caracterizar la población de esta especie, así como sus movimientos y migraciones en el PNIC y aguas adyacentes. Si conocemos la dinámica de esta población, su composición, estacionalidad y relación con variables ambientales, se podrá recomendar estrategias para su manejo sostenible en la zona de amortiguamiento AMM-MS. A su vez, esta información podría ser utilizada para prevenir futuras faenas pesqueras ilegales en el PNIC en sitios o épocas críticas.
3. El alto porcentaje de atunes encontrados con vida en las líneas de pesca hace necesario fortalecer las capacidades de los funcionarios y así mejorar la eficiencia durante sus intervenciones. Es necesario que el PNIC cuente con embarcaciones más rápidas, de mayor eslora y provistas de equipo diseñado para la liberación de organismos marinos. A su vez los funcionarios deberán capacitarse en la recolección de datos biológico-pesqueros, uso de equipo y técnicas de liberación de diferentes especies pelágicas. Esta recomendación busca tener mejores datos sobre las especies capturadas, a fin de promover su mejor manejo y conservación. Además pretende disminuir el tiempo que las líneas de pesca permanecen en las aguas protegidas del PNIC, para así aumentar el porcentaje de individuos hallados con vida en este arte de pesca.

4. El análisis del estado reproductivo y las longitudes totales promedio de los atunes (T. albacares) hallados, hace muy relevante continuar monitoreando la biología reproductiva de dicha especie, esto con el fin de identificar épocas y áreas de desove y así proteger los adultos reproductores que visitan o habitan en las aguas del PNIC y AMM-MS.

5. El alto precio del atún aleta amarilla en los mercados de todo el mundo y su abundancia en el PNIC, sin duda causará que aumente la presión pesquera dentro del AMP y alrededores en los próximos años. Por tanto recomendamos la promoción de métodos de pesca sostenible para esta especie en el AMM-MS. Artes de pesca como la "varilla larga", podrían probarse, adaptarse y promoverse en el AMM-MS. Lo anterior de la mano con alianzas estratégicas con compañías pesqueras, comerciantes y consumidores que estén dispuestos a pagar más por un atún extraído de manera sostenible.

6. El aumento en la cantidad de líneas de pesca ilegal que son halladas en el AMP del PNIC durante los meses lluviosos, hace 
necesario diseñar estrategias estacionales para contrarrestar esta problemática. Por lo anterior se recomienda duplicar los patrullajes y el personal durante los meses de mayo a octubre, meses en que la cantidad de líneas halladas y de embarcaciones avistadas aumentan en aguas del PNIC. Para afrontar esta situación, podría acondicionarse una de las embarcaciones destinadas al patrullaje para permanecer varios días en el AMP, enfocando sus esfuerzos y atención al noreste del AMP.

\section{AGRADECIMIENTOS}

Agradecemos el apoyo y el esfuerzo de todos los funcionarios del Área de Conservación Marina Isla del Coco, en particular los del Parque Nacional Isla del Coco, que cada día luchan contra la pesca ilegal para proteger este Patrimonio Natural de la Humanidad. A la vez, la investigación fue posible gracias a los fondos aportados por Organización de las Naciones Unidas para la Educación, la Ciencia y la Cultura (UNESCO), Fundación Amigos de la Isla del Coco (FAICO) y Proyecto Biodiversidad Marino Costera en Costa Rica, Desarrollo de Capacidades y Adaptación al Cambio Climático (BIOMARCC). Por último agradecer a Katherine Cure por la revisión realizada a la versión en inglés del resumen del manuscrito.

\section{RESUMEN}

El Parque Nacional Isla del Coco (PNIC), Costa Rica, sobresale por sus grandes congregaciones de especies pelágicas, en especial de tiburones (Carcharhinidae y Sphyrnidae), picudos (Istiophoridae) y atunes (Scombridae). Esta riqueza atrae embarcaciones de pesca comerciales que ingresen a las aguas protegidas del Parque Nacional. En la actualidad esta interacción entre especies pelágicas y pesquerías ilegales dentro PNIC representa una de las mayores amenazas a la integridad ecológica marina de esta isla oceánica. El PNIC invierte recursos humanos y económicos en la protección y control de sus $22.22 \mathrm{~km}$ de AMP (área marina protegida) realizando patrullajes de vigilancia. Durante el 2012, 2013 y hasta agosto 2014 se encontraron 108 líneas de pesca de flote o palangres y cerca de 13000 anzuelos dentro del AMP. En total se decomisaron $503.5 \mathrm{~km}$ de líneas de pesca, en donde se hallaron
257 organismos. La especie más capturada fue el atún aleta amarilla (Thunnus albacares), representando más del 72,3 $\%$ de toda la captura $(\mathrm{n}=186)$. Los T. albacares analizados revelaron una longitud total media de $153,35 \pm 23.19 \mathrm{~cm}$ (Min=90 cm; Max=190 cm); lo cual nos indica que la mayoría de los individuos eran adultos. Lo anterior debido a que, para $T$. albacares, las tallas de primera madurez sexual reportadas oscilan entre los 90 y $120 \mathrm{~cm}$ (Albaret, 1977; Koido, \& Suzuky, 1989). Los análisis de CPUE revelan las mayores capturas de $T$. albacares entre mayo y agosto de cada año, con una abundancia relativa promedio para el 2012 de $26.13 \pm 54.71$ atunes (T. albacares) por cada 1000 anzuelos hallados. Mientras que en 2013 la CPUE fue de $11.63 \pm 23.79$ atunes cada 1000 anzuelos y en el 2014 de $13.71 \pm 24.52$ atunes cada 1000 anzuelos.

Palabras claves: Pesca ilegal, Parque Nacional Isla del Coco, atún aleta amarilla, Thunnus albacares.

\section{REFERENCIAS}

Área de Conservación Marina Isla del Coco (ACMIC). (2006). Informes periódicos sobre la aplicación de la convención del Patrimonio Mundial. San José, Costa Rica: SINAC, MINAE.

Albaret, J. J. (1977). La reproduction de l'albacore (Thunnus albacares) dans le Golfe de Guinée. Cash. ORSTOM. Séries Océanographie, 15(4), 389-419.

Arias, A., Pressey, R. L., Jones, R. E., Álvarez-Romero, J., \& Cinner, J. E. (2014). Optimizing enforcement and compliance in offshore marine protected areas: A case study from Cocos Island, Costa Rica. Oryx, 1 . doi:10.1017/S0030605314000337.

Bessudo, S., Soler, G., Klimley, P., Ketchum, J., Hearn, A., \& Arauz, R. (2011). Residency of the scalloped hammerhead shark (Sphyrna lewini) at Malpelo Island and evidence of migration to other islands in the Eastern Tropical Pacific. Environmental Biology of Fishes, 91, 165-176.

Cajiao, V. (Ed.). (2005). Ley de Pesca y Acuicultura. San José, Costa Rica: Litografía IPECA.

Campos, J. A., Segura, A., Lozano, O., \& Madrigal, E. 1993. Ecología básica de Coryphaena hippurus (Pisces, Coryphaenidae) y abundancia de grandes pelágicos en el Pacífico de Costa Rica. Revista Biología Tropical, 41(3), 783-790.

Castro, C., Gonzáles, W., \& Estrada, F. (2008). Informe Anual: CONVENIO GUARDACOSTAS-MINAEMARVIVA Comisión tripartita. San José, Costa Rica: Ministerio de Ambiente Energía y Telecomunicaciones (MINAET).

Cortés, J. (2008). Historia de la investigación marina de la Isla del Coco, Costa Rica. Revista de Biología Tropical, 56(Suplemento 2), 1-18. 
Cortés, J. (2012). Marine biodiversity of an Eastern Tropical Pacific oceanic island, Isla del Coco, Costa Rica. Revista de Biología Tropical, 60(Suplemento 3), 131-185

Davis, M. W. (2002). Key principles for understanding fish bycatch discard mortality. Canadian Journal of Fisheries and Aquatic Sciences, 59, 1834-1843.

Di Rienzo, J. A., Casanoves, F., Balzarini, M., Gonzalez, L., Tablada, M., \& Robledo, C. (2008). InfoStat, versión 2008. Grupo InfoStat, FCA, Universidad Nacional de Córdoba, Argentina.

Gale, M. K., Hinch, S., \& Donaldson, M. R. (2013). The role of temperature in the capture and release of fish. Fish and Fisheries, 14, 1-33.

Gallagher, A. J., Orbesenc, E. S., Hammerschlaga, N., \& Serafy, J. E. (2014). Vulnerability of oceanic sharks as pelagic longline bycatch. Global Ecology and Conservation, 1, 50-59.

Halpern, B. (2003). The impact of Marine Reserves: Do reserves work and does reserve size matter? Ecological Applications, 13(1), S117-S137.

Kelleher, G., Bleakley, C., \& Wells, S. (1995). A Global representative system of Marine Protected Areas. Volume I. Washington, DC: The Great Barrier Reef Marine Authority, The World Bank and The World Conservation Union (IUCN).

Klimley, P., Jorgensen, S., Melo, A., \& Beavers, S. (2003) The occurrence of yellowfin tuna (Thunnus albacares) at Espiritu Santo Seamount in the Gulf of California. Fish Bulletin, 101, 684-692.

Knapp, J. M., Heinisch1, G., Rosenfeld, H., \& Lutcavage, M. E. (2013). New results on maturity status of Western Atlantic bluefin tuna, Thunnus thynnus. Collective Volume of Scientific Papers ICCAT, 69(2), 1005-1015.

Koido, T., \& Suzuki, Z. (1989). Main spawning season of yellowfin tuna, Thunnus albacares, in the western tropical Pacific Ocean based on the gonad index. Bulletin of the National Research Institute of Far Seas Fisheries, 26, 153-164.

Lasso, J., \& Zapata, L. (1999). Fisheries and biology of Coryphaena hippurus in the Pacific coast of
Colombia and Panama. Scientia Marina, 63(3-4), 387-399.

Lizano, O. (2008). Dinámica de aguas alrededor de la Isla del Coco, Costa Rica. Revista Biología Tropical, 56(Suplemento 2), 31-48.

Lizano, O. 2012. Rasgos morfológicos alrededor de la Isla del Coco y de sus montes submarinos vecinos, Pacífico de Costa Rica. Revista Biología Tropical, 60(Suplemento 3), 43-51.

Morato, T., Hoyle, S. D., Allain, V., \& Nicol, S. L. (2010). Seamounts are hotspots of pelagic biodiversity in the open ocean. Proceedings of the National of Sciences of the United States of America, 107, 9707-9711.

Patterson, K. R., \& Martínez, J. (1991). Exploitation of dolphin-fish Coryphaena hipurrus off Ecuador: Analysis by length-based virtual population analysis. Fish byte, 9(2), 21-23.

Pauly, D., Christensen, V., Guénette, S., Pitcher, T. J., Sumaila, R. U., Walters, C. J., Watson, R., \& Zeller, D. (2002). Towards sustainability in world fisheries. Nature, $418,8$.

Roberts, C. M., Hawkins, J. P., \& Gell, F. R. (2005). The role of marine reserves in achieving sustainable fisheries. Philosophical Transactions of the Royal Society B: Biological Sciences, 360, 123-132.

Serna, J. (2006). Caracterización de las embarcaciones registradas en el Parque Nacional Isla del Coco. (Informe Técnico Proyecto-Protección de la Biodiversidad del Área de Conservación Marina Isla del Coco (ACMIC)). San José, Costa Rica: Fondo Francés para el Medio Ambiente Mundial (FFMEM) y SINAC.

Swimmer, Y., Wang, J., Arauz, R., \& McCracken, M. (2010). Effects of hook offsets on target species catch rates and sea turtle bycatch in a shallow Costa Rican longline fishery. Aquatic Conservation of Marine and Freshwater Ecosystems, 10, 245-254.

Swimmer, Y., Suter, J., Arauz, R., Bigelow, K., López, A., Zanella, I., Bolaños, A., Ballestero, J., Suarez, R., Wang, J., \& Boggs, C. (2011). Sustainable fishing gear: the case of modified circle hooks in a Costa Rican longline fishery. Marine Biology, 158, 757-767. 
